VOL. 45 (1992) [453-462]

\title{
ON POLYGONAL PRODUCTS OF FINITELY GENERATED ABELIAN GROUPS
}

\author{
GoANSU KIM
}

\begin{abstract}
We prove that a polygonal product of polycyclic-by-finite groups amalgamating subgroups, with trivial intersections, is cyclic subgroup separable (hence, it is residually finite) if the amalgamated subgroups are contained in the centres of the vertex groups containing them. Hence a polygonal product of finitely generated abelian groups, amalgamating any subgroups with trivial intersections, is cyclic subgroup separable. Unlike this result, most polygonal products of four finitely generated abelian groups, with trivial intersections, are not subgroup separable $(\mathcal{L E R \mathcal { F }})$. We find necessary and sufficient conditions for certain polygonal products of four groups to be subgroup separable.
\end{abstract}

\section{INTRODUCTION}

Polygonal products of groups were introduced by Karrass, Pietrowski and Solitar [6]. Using their result, Brunner, Frame, Lee and Wielenberg [5] determined all torsionfree subgroups of finite index in the Picard group $P S L(2, Z[i])$. In [3], Allenby and Tang proved that polygonal products of four finitely generated free abelian groups, amalgamating cyclic subgroups with trivial intersections, is residually finite. Kim and Tang [9] showed that certain polygonal products of four nilpotent groups, amalgamating cyclic subgroups with trivial intersections, are residually finite. In this paper, we prove that polygonal products of more than four polycyclic-by-finite groups amalgamating any subgroups, contained in the centres of their vertex groups, with trivial intersections are $\pi_{c}$ (Theorem 2.11), hence they are residually finite. Thus, polygonal products of more than four finitely generated abelian groups, amalgamating any subgroups with trivial intersections, are $\pi_{c}$. It was relatively easy to prove the same result for those polygonal products with four vertex groups and cyclic subgroups amalgamated [8]. Note that polygonal products of four polycyclic-by-finite groups amalgamating cyclic subgroups, contained in the centres of their vertex groups, with trivial intersections is conjugacy separable [7]. Unlike the case for residual finiteness or for conjugacy separability, most

Received 28 May 1991

This article is condensed from the author's Ph.D. thesis, submitted to the University of Waterloo, Canada.

Copyright Clearance Centre, Inc. Serial-fee code: 0004-9729/92 \$A2.00+0.00. 
polygonal products of four finitely generated abelian groups amalgamating cyclic subgroups with trivial intersections are not subgroup separable (Theorem 3.3). We also find necessary and sufficient conditions for certain polygonal products of four groups to be subgroup separable (Theorem 3.2).

Briefly polygonal products of groups can be described as follows [3]: Let $P$ be a polygon. Assign a group $G_{v}$ to each vertex $v$ and a group $G_{e}$ to each edge $e$ of $P$. Let $\alpha_{e}$ and $\beta_{e}$ be monomorphisms which embed $G_{e}$ as a subgroup of the two vertex groups at the ends of the edge $e$. Then the polygonal product $G$ is defined to be the group generated by the generators and relations of the vertex groups together with the extra relations obtained by identifying $g_{e} \alpha_{e}$ and $g_{e} \beta_{e}$ for each $g_{e} \in G_{e}$.

By abuse of language, we say that $G$ is the polygonal product of the (vertex) groups $G_{0}, G_{1}, \ldots, G_{n}$, amalgamating the (edge) subgroups $H_{0}, H_{1}, \ldots, H_{n}$ with trivial intersections, if $G_{i} \cap G_{i+1}=H_{i}$ and $H_{i} \cap H_{i+1}=1$, where $0 \leqslant i \leqslant n$ and the subscripts $i$ are taken modulo $n+1$.

Finally, we note that a polygonal product can appear as a subgroup of a group, and then the residual properties of the polygonal product determine the residual properties of the whole group, as in the following example.

EXAMPLE 1.1: Let $G=\left\langle a, b ;\left[a, b^{-1} a b\right], a^{m}, b^{n}\right\rangle$, where $n \neq 0$. Clearly $G$ is a finite cyclic extension of $\langle a\rangle^{G}$. We note that $\langle a\rangle^{G}=\left\langle a_{0}, a_{1}, \ldots, a_{n-1} ; a_{i}^{m},\left[a_{i}, a_{i+1}\right]\right\rangle$, where $a_{i}=b^{-i} a b^{i}$ and the subscripts $i$ are considered modulo $n$. For $n \geqslant 3$, we may consider $\langle a\rangle^{G}$ as the polygonal product of the abelian subgroups $\left\langle a_{i}, a_{i+1}\right\rangle$ amalgamating the subgroups $\left\langle a_{i+1}\right\rangle$, where the subscripts are taken modulo $n$. By Theorem 2.12 , we can see that $\langle a\rangle^{G}$ is $\pi_{c}$ for $n \geqslant 4$. If $n=1,2,3$ then $\langle a\rangle^{G}$ is finite abelian. Therefore, $G$ is $\pi_{c}$ for all $n \neq 0$.

We shall adopt the following notation and terminology:

We use $N \triangleleft_{f} G$ to denote that the normal subgroup $N$ of $G$ has finite index in $G$ and "f.g." means "finitely generated". We denote by $A *_{H} B$ the generalised free product of $A$ and $B$ with the subgroup $H$ amalgamated. If $G=A *_{H} B$ and $x \in G$, then $\|x\|$ denotes the free product length of $x$ in $G$. On the other hand, we use $|x|$ to denote the order of $x$. If $\bar{G}$ is a homomorphic image of $G$, then we use $\bar{x}$ to denote the image of $x \in G$ in $\bar{G}$.

Let $H$ be a subgroup of a group $G$. Then $G$ is said to be $H$-separable if, for each $x \in G \backslash H$, there exists $N \triangleleft_{f} G$ such that $x \notin N H$. A group $G$ is locally extended residually finite ( $\mathcal{L} \mathcal{E} \mathcal{R} \mathcal{F}$ or subgroup separable) if $G$ is $H$-separable for all f.g. subgroups $H$ of $G$.

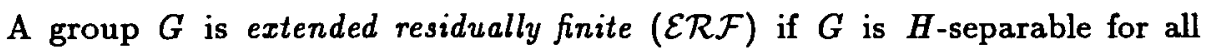
subgroups $H$ of $G$.

A group $G$ is cyclic subgroup separable $\left(\pi_{c}\right)$ if $G$ is $\langle x\rangle$-separable for all 
$x \in G$.

A group $G$ is residually finite $(\mathcal{R} \mathcal{F})$ if $G$ is $\langle 1\rangle$-separable.

Clearly, every $\mathcal{L E R \mathcal { F }}$ group is $\pi_{c}$, and every $\pi_{c}$ group is $\mathcal{R F}$.

We shall use the following results:

ThEOREM 1.2. [4], [1] If $A$ and $B$ are $\mathcal{R F}\left(\pi_{c}, \mathcal{L E R F}\right)$ and $U$ is finite, then $A *_{U} B$ is $\mathcal{R F}\left(\pi_{c}, \mathcal{L E R \mathcal { F }}\right.$, respectively).

THEOREM 1.3. [1] Let $G=N \cdot H$ be a split extension of the normal f.g. subgroup $N$ by $B$. If $N$ is $\mathcal{E R \mathcal { F }}$ and $H$ is $(\mathcal{L}) \mathcal{E R F}$, then $G$ is $(\mathcal{L}) \mathcal{E R \mathcal { F }}$. If $N$ and $H$ are both $\pi_{c}$ groups, then $G$ is a $\pi_{c}$ group.

THEOREM 1.4. [8] Let $G=E *_{H} F$. Suppose that

(a) $E$ and $F$ are $\pi_{c}$ and $H$-separable,

(b) for each $N \triangleleft_{f} H$ there exist $N_{E} \triangleleft_{f} E$ and $N_{F} \triangleleft_{f} F$ such that $N_{E} \cap H=$ $N_{F} \cap H \subset N$.

Then $G$ is $\pi_{\mathrm{c}}$.

A group $G$ is polycyclic-by-finite if it has a normal subgroup $N$ such that $N$ is polycyclic and $G / N$ is finite.

\section{CYCliC SUbgroup SEPARABILITY $\left(\pi_{c}\right)$}

A group $G$ is polycyclic-by-finite if it has a normal subgroup $N$ such that $N$ is polycyclic and $G / N$ is finite.

In this section we shall prove that a polygonal product of polycyclic-by-finite groups $A_{0}, A_{1}, \ldots, A_{n}(n \geqslant 3)$, amalgamating any subgroups $H_{0}, H_{1}, \ldots, H_{n}$ with trivial intersections, is $\pi_{c}$ if $H_{i-1}, H_{i} \subset Z\left(A_{i}\right)$ for all $i$. To prove this result, we have to study some properties of the group $E_{m}=A_{1} *_{H_{1}} A_{2} *_{H_{2}} \cdots *_{H_{m-1}} A_{m}$, where $H_{j}=A_{j} \cap A_{j+1}$ for $1 \leqslant j \leqslant m-1$, and each $A_{i}$ is a polycyclic-by-finite group containing subgroups $H_{i-1}$ and $H_{i}$ such that $H_{i-1} \cap H_{i}=1$, where $H_{i-1}, H_{i} \subset Z\left(A_{i}\right)$ for $1 \leqslant i \leqslant m$. Throughout this section $E_{m}$ denotes the above group.

Lemma 2.1. For given subgroups $U \triangleleft_{f} H_{0}$ and $V \triangleleft_{f} H_{m}$, there exists $N \triangleleft_{f}$ $E_{m}$ such that $N \cap H_{0}=U, N \cap H_{m}=V$ and $N H_{0} \cap N H_{m}=N$.

Proof: CASE 1: $m>1$. There exists a homomorphism $\pi: E_{m} \rightarrow\left(A_{1} / H_{1} U\right) *$ $\left(A_{m} / H_{m-1} V\right)$, since $H_{i-1}, H_{i} \subset Z\left(A_{i}\right)$. Now $A_{1} / H_{1} U$ and $A_{m} / H_{m-1} V$ are polycyclic-by-finite, hence, $\bar{E}_{m}=E_{m} \pi$ is $\mathcal{R F}$. Thus, since $\bar{H}_{0}=H_{0} H_{1} U / H_{1} U \cong$ $H_{0} / U$ and $\bar{H}_{m} \cong H_{m} / V$ are finite, there exists $\bar{N} \triangleleft_{f} \bar{E}_{m}$ such that $1=\bar{N} \cap \overline{H_{m} H_{0}}$. Let $N$ be the preimage of $\bar{N}$ in $E_{m}$.

CASE 2. $m=1$. Let $U \triangleleft_{f} H_{0}$ and $V \triangleleft_{f} H_{1}$ be given. Since $U, V \subset Z\left(A_{1}\right)$, we can consider $\bar{A}_{1}=A_{1} / U V$. Then the subgroups $\bar{H}_{0}$ and $\bar{H}_{1}$ of $\bar{A}_{1}$ are finite. Since $\bar{A}_{1}$ is 
$\mathcal{R F}$, there exists $\bar{N} \triangleleft_{f} \bar{A}_{1}$ such that $1=\bar{N} \cap \overline{H_{1} \bar{H}_{0}}$. Let $N$ be the preimage of $\bar{N}$ in $A_{1}$.

It is not difficult to see that $N$ satisfies our requirements.

Lemma 2.2. For any given $M \triangleleft_{f} E_{n-1}$ and $N \triangleleft_{f} A_{n}$, there exist $P \triangleleft_{f} E_{n-1}$ and $Q \triangleleft_{f} A_{n}$ such that $P \subset M, Q \subset N, P \cap H_{n-1}=Q \cap H_{n-1}, P H_{0} \cap P H_{n-1}=P$ and $Q H_{n-1} \cap Q H_{n}=Q$.

Proof: By Lemma 2.1, there exists $M_{1} \triangleleft_{f} E_{n-1}$ such that $M_{1} \cap H_{0}=M \cap H_{0}$, $M_{1} \cap B_{n-1}=M \cap N$, and $M_{1} H_{0} \cap M_{1} H_{n-1}=M_{1}$. Similarly, there exists $N_{1} \triangleleft_{f} A_{n}$ such that $N_{1} \cap H_{n-1}=M \cap N, N_{1} \cap H_{n}=N \cap H_{n}$, and $N_{1} H_{n-1} \cap N_{1} H_{n}=N_{1}$. Let $P=M \cap M_{1}$ and $Q=N \cap N_{1}$. Then it is easy to see that $P$ and $Q$ satisfy the required conditions.

Theorem 2.3. Let $G=E *_{H} F$, where $E, F$ are $H$-separable. Let $S$ be a subgroup of $E$ and suppose that $E$ is $S$-separable. Suppose, further, that

(W) for each $N \triangleleft_{f} B$ there exist $N_{E} \triangleleft_{f} E$ and $N_{F} \triangleleft_{f} F$ such that $N_{E} \cap H=$ $N_{F} \cap H \subset N$.

Then $G$ is $S$-separable.

Proof: Let $g \in G$ be such that $g \notin S$.

CASE 1. $g \in E$. Since $E$ is $S$-separable, there exists $P \triangleleft_{f} E$ such that $g \notin P S$. Now, by (W), there exist $P_{1} \triangleleft_{f} E$ and $Q_{1} \triangleleft_{f} F$ such that $P_{1} \cap H=Q_{1} \cap H \subset P \cap H$. Let $N_{E}=P \cap P_{1}$ and $N_{F}=Q_{1}$. Then $N_{E} \triangleleft_{f} E, N_{F} \triangleleft_{f} F$, and $N_{E} \cap H=P_{1} \cap H=$ $Q_{1} \cap H=N_{F} \cap H$. Thus, we have a homomorphism $\pi: E *_{H} F \rightarrow E / N_{E} *_{\overparen{H}} F / N_{F}$, where $\bar{H}=H N_{E} / N_{E}=H N_{F} / N_{F}$. It is clear that $\bar{g} \notin \bar{S}$, where $\bar{G}=G \pi$. Since $\bar{G}$ is $\mathcal{L} \mathcal{E R F}$ by Theorem 1.2 , there exists $\bar{M} \triangleleft_{f} \bar{G}$ such that $\bar{g} \notin \overline{M S}$. Let $M$ be the preimage of $\bar{M}$ in $G$. Then, clearly, $M \triangleleft_{f} G$ and $g \notin M S$.

CASE 2. $g \in F \backslash H$. Since $F$ is $H$-separable, we can find $Q \triangleleft_{f} F$ such that $g \notin Q H$. By (W), there exist $P_{1} \triangleleft_{f} E$ and $Q_{1} \triangleleft_{f} F$ such that $P_{1} \cap H=Q_{1} \cap H \subset Q \cap H$. Let $N_{E}=P_{1}$ and $N_{F}=Q \cap Q_{1}$. Then $N_{E} \triangleleft_{f} E, N_{F} \triangleleft_{f} F$, and $N_{E} \cap H=P_{1} \cap H=$ $Q_{1} \cap H=N_{F} \cap H$. Now we consider $\pi: G \rightarrow E / N_{E} *_{\bar{H}} F / N_{F}$ as in Case 1. Then $\bar{g} \in \bar{F} \backslash \bar{H}$ and $\bar{S} \subset \bar{E}$. It follows that $\bar{g} \notin \bar{S}$. As in Case 1, we can find $M \triangleleft_{f} G$ such that $g \notin M S$.

CASE 3. $\|g\| \geqslant 2$. Assume that $g=e_{1} f_{1} \cdots e_{n} f_{n}$, where $e_{i} \in E \backslash H$ and $f_{i} \in F \backslash H$ (the other cases are similar). Since $E$ and $F$ are $H$-separable, there exist $P \triangleleft_{f} E$ and $Q \triangleleft_{f} F$ such that $e_{i} \notin P H$ and $f_{i} \notin Q H$ for all $i$. Considering $P \cap Q \triangleleft_{f} H$, by assumption (W), we can find $P_{1} \triangleleft_{f} E$ and $Q_{1} \triangleleft_{f} F$ such that $P_{1} \cap H=Q_{1} \cap H \subset P \cap Q$. Let $N_{E}=P \cap P_{1}$ and $N_{F}=Q \cap Q_{1}$. Then $N_{E} \triangleleft_{f} E, N_{F} \triangleleft_{f} F$ and $N_{E} \cap H=N_{F} \cap H$. Thus we have a homomorphism $\pi: G \rightarrow E / N_{E} *_{\bar{H}} F / N_{F}$ as in Case 1. Then we have 
$\|\bar{g}\|=\|g\| \geqslant 2$, where $\bar{G}=G \pi$. It follows that $\bar{g} \notin \bar{S}$. Now, as in Case 1 , we can find $M \triangleleft_{f} G$ such that $g \notin M S$. This proves the theorem.

If $S=\langle 1\rangle$, then Theorem 2.3 proves that $G=E *_{H} F$ is $\mathcal{R F}$. Thus Theorem 2.3 is a generalised version of [11, Criterion] or [2, Lemma 3.1]. Furthermore, using the above theorem, we can prove the following two results which are unavoidable for our main result in this section.

COROLlaRY 2.4. The group $E_{n}$ is $H_{0}$-separable and $H_{n}$-separable.

Proof: Note that $E_{1}$ and $E_{2}$ are $\mathcal{L E R F}$ by [1, Theorem 5]. Hence the lemma holds for $n=1,2$. Inductively, we assume that $E_{n-1}$ is $H_{0}$-separable and $H_{n-1^{-}}$ separable. Note that the $A_{i}$ are polycyclic-by-finite, hence $A_{n}$ is also $H_{n-1}$-separable. By Lemma 2.1, for each $N_{H} \triangleleft_{f} H_{n-1}$, there exist $N \triangleleft_{f} E_{n-1}$ and $M \triangleleft_{f} A_{n}$ such that $N \cap H_{n-1}=N_{H}=M \cap H_{n-1}$. This proves (W) in Theorem 2.3 for $E_{n}=E_{n-1} *_{H_{n-1}} A_{n}$. Thus $E_{n}$ is $H_{0}$-separable by Theorem 2.3. By symmetry, $E_{n}$ is $H_{n}$-separable.

Corollary 2.5. The group $E_{n}$ is $\pi_{c}$.

PRoof: Write $E_{n}=E_{n-1} *_{H_{n-1}} A_{n}$. Then $E_{n-1}$ and $A_{n}$ are $H_{n-1}$-separable (Corollary 2.4) and satisfy (W) in Theorem 2.3 as in the previous proof. It follows, by induction and Theorem 1.4, that $E_{n}$ is $\pi_{c}$.

Lemma 2.6. For given $x \in E_{m}$ such that $x \notin B_{0} H_{m}$, there exists $N \triangleleft_{f} E_{m}$ such that $x \notin N H_{0} H_{m}$.

Proof: For $m=1$, the lemma is trivial, since $E_{1}=A_{1}$ is polycyclic-by-finite (hence, it is $\mathcal{L E R \mathcal { F }}$ ) and since $\boldsymbol{H}_{0} \boldsymbol{H}_{1}$ is a $\mathrm{f}$.g. subgroup. For an induction, we assume that the lemma holds for $E_{m-1}$; that is, for given $e \in E_{m-1}$ such that $e \notin H_{0} H_{m-1}$, there exists $P \triangleleft_{f} E_{m-1}$ such that $e \notin P H_{0} H_{m-1}$. We consider $E_{m}=E_{m-1} *_{H_{m-1}}$ $A_{m}$.

CASE 1. Suppose that $x \notin H_{0} H_{m}$ is implied by the syllable length of $x$; that is,

(1) $\|x\| \geqslant 3$; or,

(2) $\|x\|=2$ and $x \in A_{m} E_{m-1}$.

Consider the case $x=e_{1} a_{1} \cdots e_{n} a_{n}$, where $e_{i} \in E_{m-1} \backslash H_{m-1}$ and $a_{i} \in A_{m} \backslash H_{m-1}$ (the other cases are similar). Since $E_{m-1}, A_{m}$ are $H_{m-1}$-separable by Corollary 2.4 , there exist $P_{1} \triangleleft_{f} E_{m-1}$ and $Q_{1} \triangleleft_{f} A_{m}$ such that $e_{i} \notin P_{1} H_{m-1}$ and $a_{i} \notin Q_{1} H_{m-1}$, for all $i$. Now, by Lemma 2.2, there exist $P \triangleleft_{f} E_{m-1}$ and $Q \triangleleft_{f} A_{m}$ such that $P \subset P_{1}$, $Q \subset Q_{1}, P \cap H_{m-1}=Q \cap H_{m-1}, P H_{0} \cap P H_{m-1}=P$, and $Q H_{m-1} \cap Q H_{m}=Q$. Hence, considering the natural homomorphism $\pi: E_{m} \rightarrow\left(E_{m-1} / P\right) * \bar{H}_{m-1}\left(A_{m} / Q\right)$, where $\bar{H}_{m-1}=H_{m-1} P / P=H_{m-1} Q / Q$, we have $\|\bar{x}\|=\|x\|$ and $\bar{H}_{0} \cap \bar{H}_{m-1}=\langle 1\rangle=$ $\bar{H}_{m-1} \cap \bar{H}_{m}$. Then clearly $\bar{x} \notin \overline{H_{0} H_{m}}$. 
CASE 2. $\|x\|=2$ and $x \notin A_{m} E_{m-1}$; that is, $x=e a$ where $e \in E_{m-1} \backslash H_{m-1}$ and $a \in A_{m} \backslash H_{m-1}$. Thus, by Corollary 2.4, there exist $P_{1} \triangleleft_{f} E_{m-1}$ and $Q_{1} \triangleleft_{f} A_{m}$ such that $e \notin P_{1} H_{m-1}$ and $a \notin Q_{1} H_{m-1}$. We note that $x=e a \notin H_{0} H_{m}$ if, and only if, one of the following is true:

(1) $e \notin H_{0} H_{m-1}$; or

(2) $e=h_{1} r$ and $r a \notin H_{m}$, where $h_{1} \in H_{0}$ and $r \in H_{m-1}$.

If (1) is true then, by the induction hypothesis, there exists $P_{2} \triangleleft_{f} E_{m-1}$ such that $e \notin P_{2} H_{0} H_{m-1}$. Then, by Lemma 2.2, there exist $P \triangleleft_{f} E_{m-1}$ and $Q \triangleleft_{f} A_{m}$ such that $P \subset P_{1} \cap P_{2}, Q \subset Q_{1}, P \cap H_{m-1}=Q \cap H_{m-1}, P H_{0} \cap P H_{m-1}=P$, and $Q H_{m-1} \cap Q H_{m}=Q$. Consider the homomorphism $\pi: E_{m} \rightarrow E_{m-1} / P *_{\bar{H}_{m-1}} A_{m} / Q$, as above. Note that $\bar{H}_{0} \cap \bar{H}_{m-1}=1=\bar{H}_{m-1} \cap \bar{H}_{m}, \bar{x}=\overline{e a}$ and $\bar{e} \notin \bar{H}_{0} \bar{H}_{m-1}$. It follows that $\bar{x} \notin \overline{H_{0} H_{m}}$.

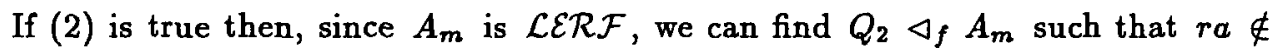
$Q_{2} H_{m}$. As before, we can find $P \triangleleft_{f} E_{m-1}$ and $Q \triangleleft_{f} A_{m}$ such that $P \subset P_{1}$, $Q \subset Q_{1} \cap Q_{2}, P \cap H_{m-1}=Q \cap H_{m-1}, P H_{0} \cap P H_{m-1}=P$, and $Q H_{m-1} \cap Q H_{m}=Q$. Then, as before, we have $\bar{x}=\overline{e a} \notin \overline{H_{0} H_{m}}$, where $\overline{E_{m}}=E_{m} \pi$.

CASE 3. $\|x\|=1$. Consider the case $x \in E_{m-1} \backslash H_{m-1}$ (the other case being similar). Since $x \notin H_{0}$, there exists $P_{1} \triangleleft_{f} E_{m-1}$ such that $x \notin P_{1} H_{0} \cup P_{1} H_{m-1}$. Now, by Lemma 2.2, there exist $P \triangleleft_{f} E_{m-1}$ and $Q \triangleleft_{f} A_{m}$ such that $P \subset P_{1}, P \cap H_{m-1}=$ $Q \cap H_{m-1}, P H_{0} \cap P H_{m-1}=P$, and $Q H_{m-1} \cap Q H_{m}=Q$. Then, we can easily see that $\bar{x} \notin \overline{H_{0} \bar{H}_{m}}$, where $\bar{E}_{m}=E_{m} \pi$ as above.

CASE 4. $\|x\|=0$. In this case we have $x \in H_{m-1}$ and $x \neq 1$. Since $E_{m-1}$ is $\mathcal{R F}$ (Corollary 2.5), there exists $P_{1} \triangleleft_{f} E_{m-1}$ such that $x \notin P_{1}$. As in Case 3, we can find $P \triangleleft_{f} E_{m-1}$ and $Q \triangleleft_{f} A_{m}$ such that $\bar{x} \notin \overline{H_{0} \bar{H}_{m}}$, where $\bar{E}_{m}=E_{m} \pi$.

Consequently, we have found $P \triangleleft_{f} E_{m-1}$ and $Q \triangleleft_{f} A_{m}$ such that $\bar{x} \notin \overline{H_{0} H_{m}}$, where $\bar{E}_{m}=E_{m} \pi=E_{m-1} / P *_{H_{m-1}} A_{m} / Q$. Since $\left|\bar{H}_{0}\right|$ and $\left|\bar{H}_{m}\right|$ are finite, and since $\bar{E}_{m}$ is $\mathcal{R F}$, it is not difficult to find $\bar{N} \triangleleft_{f} \bar{E}_{m}$ such that $\bar{x} \notin \overline{N H_{0} H_{m}}$. Let $N$ be the preimage of $\bar{N}$ in $E_{m}$. Then clearly, $N \triangleleft_{f} E_{m}$ and $x \notin N H_{0} H_{m}$ as required.

Definition 2.7: [9] Let $G=G_{1} *_{H} G_{2}$. Let $X, Y$ be subgroups of $G_{1}, G_{2}$ respectively. Let $\mathcal{N}=\left\{\left(N_{i}, M_{i}\right) ; i \in I\right\}$ be a collection of pairs of normal subgroups of $G_{1}$ and $G_{2}$ satisfying the following:

(1) $N_{i} \triangleleft G_{1}, M_{i} \triangleleft G_{2}$, and $N_{i} \cap H=M_{i} \cap H$, for all $i \in I$.

(2) $N_{i} \cap X H=\left(N_{i} \cap X\right)\left(N_{i} \cap H\right)$ and $M_{i} \cap Y H=\left(M_{i} \cap Y\right)\left(M_{i} \cap H\right)$, for all $i \in I$,

(3) $\left(\bigcap_{j=1}^{n} N_{\alpha_{j}}, \bigcap_{j=1}^{n} M_{\alpha_{j}}\right) \in \mathcal{N}$ for all $\alpha_{1}, \ldots, \alpha_{n} \in I$, where $n$ is finite, 
(4) $\bigcap_{i \in I} N_{i} X=X, \bigcap_{i \in I} N_{i} H=H, \bigcap_{i \in I} M_{i} Y=Y$, and $\bigcap_{i \in I} M_{i} H=H$,
(5) $\bigcap_{i \in I} N_{i} X H=X H$ and $\bigcap_{i \in I} M_{i} Y H=Y H$.

Then $\mathcal{N}$ is called a compatible filter of $G$ with respect to the subgroups $X$ and $Y$.

Lemma 2.8. [9] Let $G=G_{1} *_{H} G_{2}$. Let $X, Y$ be subgroups of $G_{1}, G_{2}$ respectively, such that $X \cap B=Y \cap H=1$. Let $\mathcal{N}$ be a compatible filter of $G$ with respect to $X$ and $Y$. Then, for each $g \in G \backslash(X * Y)$ with $\|g\| \geqslant 1$, there exists $(N, M) \in \mathcal{N}$ such that $\|g \pi\|=\|g\|$ and $g \pi \notin X \pi * Y \pi$, where $\pi$ is the canonical homomorphism of $G$ onto $\bar{G}=\bar{G}_{1}{ }_{\bar{H}} \bar{G}_{2}$, and where $\bar{G}_{1}=G_{1} / N, \bar{G}_{2}=G_{2} / M$ and $\bar{H}=H N / N=H M / M$.

For example, we can see, by Corollary 2.4 and Lemma 2.6 together with Lemma 2.2, that $\mathcal{N}=\left\{(P, Q): P \triangleleft_{f} E_{m-1}, Q \triangleleft_{f} A_{m}, P \cap H_{m-1}=Q \cap H_{m-1}, P H_{0} \cap P H_{m-1}=\right.$ $\left.P, Q H_{m-1} \cap Q H_{m}=Q\right\}$ is a compatible filter of $E_{m}=E_{m-1} *_{H_{m-1}} A_{m}$ with respect to $H_{0}$ and $H_{m}$.

THEOREM 2.9. Let $G=G_{1} *_{H} G_{2}$, and let $X<G_{1}, Y<G_{2}$ be such that $X \cap H=1=Y \cap H$. Suppose that $G$ has a compatible filter $\mathcal{N}=\left\{\left(N_{i}, M_{i}\right): i \in I\right\}$ of $G$ with respect to $X$ and $Y$, where $N_{i} \triangleleft_{f} G_{1}$ and $M_{i} \triangleleft_{f} G_{2}$, for all $i$, and suppose further that

(W') for each $N_{H} \triangleleft_{f} H$ there exists $\left(N_{j}, M_{j}\right) \in \mathcal{N}$ such that $M_{j} \cap B=$ $N_{j} \cap H \subset N_{H}$ for some $j \in I$.

Then $G$ is $X * Y$-separable whenever $H$ is $\mathcal{R F}$.

Proof: Let $g \in G \backslash(X * Y)$.

CASE 1. $g \in H$. Since $g \neq 1$ and $H$ is $\mathcal{R F}$, there exists $N_{H} \triangleleft_{f} B$ such that $g \notin N_{H}$. By $\left(\mathrm{W}^{\prime}\right)$, there exists $\left(N_{j}, M_{j}\right) \in \mathcal{N}$ such that $N_{j} \cap H=M_{j} \cap H \subset N_{H}$ for some $j \in I$. Then, $\bar{g} \notin \bar{X} * \bar{Y}$ where $\bar{G}=G_{1} / N_{j} *_{\bar{H}} G_{2} / M_{j}$ and $\bar{H}=N_{j} H / N_{j}=M_{j} H / M_{j}$. Now, $\bar{G}$ is $\mathcal{L E R \mathcal { F }}$ by Theorem 1.2 . Hence, there exists $\bar{N} \triangleleft_{f} \bar{G}$ such that $\bar{g} \notin \bar{N}(\bar{X} * \bar{Y})$. Let $N$ be the preimage of $\bar{N}$ in $G$. Then $N \triangleleft_{f} G$ and $g \notin N(X * Y)$.

CASE 2. $g \notin H$. By Lemma 2.8, there exists $\left(N_{j}, M_{j}\right) \in \mathcal{N}$ such that $\bar{g} \notin \bar{X} * \bar{Y}$, where $\bar{G}=G_{1} / N_{j} *_{\bar{H}} G_{2} / M_{j}$. Then, as before, we can find $N \triangleleft_{f} G$ such that $g \notin N(X * Y)$.

Lemma 2.10. For each $N \triangleleft_{f} H_{0} * H_{m}(m \geqslant 2)$, there exists $N_{E_{m}} \triangleleft_{f} E_{m}$ such that $N_{E_{m}} \cap\left(H_{0} * H_{m}\right)=N$.

Proof: There exists a natural homomorphism $\pi: E_{m} \rightarrow A_{1} / H_{1} * A_{m} / H_{m-1}$, obtained by defining $z \pi=1$, for all $z \in A_{2} \cup \cdots \cup A_{m-1}$, if $m \geqslant 3$; or $z \pi=1$, for all $z \in H_{1}$, if $m=2$. Let $\bar{E}_{m}=E_{m} \pi=\bar{A}_{1} * \bar{A}_{m}$, where $\bar{A}_{1}=A_{1} / H_{1}$ and 
$\bar{A}_{m}=A_{m} / H_{m-1}$. We note that $\bar{H}_{0} \cong H_{0}, \bar{H}_{m} \cong H_{m}$ and $N \cong \bar{N} \triangleleft_{f} \bar{H}_{0} * \bar{H}_{m}$. Now, considering $\bar{A}_{1} * \bar{A}_{m}=\bar{A}_{1} *_{\bar{H}_{0}}\left(\bar{H}_{0} * \bar{H}_{m}\right) * \bar{H}_{m} \bar{A}_{m}$, we have a homomorphism

$$
\phi: \bar{E}_{m} \rightarrow\left(\bar{A}_{1} / \bar{N} \cap \bar{H}_{0}\right) * \widetilde{H}_{0}\left(\bar{H}_{0} * \bar{H}_{m} / \bar{N}\right) * \widetilde{H}_{m}\left(\bar{A}_{m} / \bar{N} \cap \bar{H}_{m}\right),
$$

where $\widetilde{H}_{0}=\bar{H}_{0} / \bar{N} \cap \bar{H}_{0}=\overline{H_{0} N} / \bar{N}$ and $\widetilde{H}_{m}=\overline{H_{m} N} / \bar{N}=\bar{H}_{m} / \bar{N} \cap \bar{H}_{m}$. Since $\widetilde{H}_{0}$ and $\widetilde{H}_{m}$ are finite, therefore, $\bar{E}_{m} \phi$ is $\mathcal{R F}$. Note that $\left(\bar{H}_{0} * \bar{B}_{m}\right) / \bar{N}$ is finite. It follows that there exists $\widetilde{M} \triangleleft_{f} \bar{E}_{m} \phi$ such that $\widetilde{M} \cap\left(\left(\bar{H}_{0} * \bar{H}_{m}\right) / \bar{N}\right)=1$. Now, let $N_{E_{m}}$ be the preimage of $\widetilde{M}$ in $E_{m}$ under the homomorphism $\pi \circ \phi$. Then $N_{E_{m}} \triangleleft_{f} E_{m}$ and $N_{E_{m}} \cap\left(H_{0} * H_{m}\right)=N$ as required.

Now we are ready to show our main result of this section.

TheOREM 2.11. Let $G$ be the polygonal product of the polycyclic-by-finite groups $A_{0}, A_{1}, \ldots, A_{n}(n \geqslant 3)$, amalgamating any subgroups $H_{0}, H_{1}, \ldots, H_{n}$, with trivial intersections, where $H_{i} \subset Z\left(A_{i}\right) \cap Z\left(A_{i+1}\right)$ for all $i$, and where subscripts are taken modulo $n+1$. Then $G$ is $\pi_{c}$.

Proof: We write $G=E *_{H} F$, where $E=A_{1} *_{H_{1}} \cdots *_{H_{n-2}} A_{n-1}, F=A_{0} *_{H_{n}} A_{n}$, and $H=H_{0} * H_{n-1}$. With $G$ in this form, we can apply Theorem 1.4. For condition (a) in the theorem, Corollary 2.5 proves that $E$ and $F$ are $\pi_{c}$. Theorem 2.9, using Lemma 2.1, proves that $E$ and $F$ are $H$-separable. Also, Lemma 2.10 proves condition (b) in the theorem. Hence, by Theorem 1.4, $G$ is $\pi_{c}$.

We immediately have the following result:

Theorem 2.12. Let $G$ be the polygonal product of the f.g. abelian groups $A_{0}, A_{1}, \ldots, A_{n}(n \geqslant 3)$, amalgamating any subgroups $H_{0}, H_{1}, \ldots, H_{n}$, with trivial intersections. Then $G$ is $\pi_{c}$.

We note that the above two results are generalisations of Theorem 3.4 in [3].

\section{SUbGroup Separability $(\mathcal{L E} \mathcal{R} \mathcal{F})$}

Now we consider the subgroup separability of polygonal products of f.g. abelian groups. Throughout this section we assume that the amalgamated subgroups of polygonal products are not trivial.

Lemma 3.1. [1] If a group $G$ contains a subgroup $F_{2} \times F_{2}$, where $F_{2}$ is a free group of rank 2 , then $G$ is not $\mathcal{L E R \mathcal { F }}$.

ThEOREM 3.2. Let $P$ be the polygonal product of the four $\mathcal{L} \mathcal{E R} \mathcal{F}$ groups $A$, $B, C, D$, amalgamating the finite subgroups $H_{2}, H_{3}, H_{4}, H_{1}$, with trivial intersections, where the $H_{i}$ are contained in the centres of the vertex groups containing them. Then $P$ is $\mathcal{L E R \mathcal { F }}$ if, and only if, either $\left|H_{1}\right|=\left|H_{3}\right|=2$ or $\left|H_{2}\right|=\left|H_{4}\right|=2$. 
Proof: Let $P_{0}$ be the polygonal product of $A_{0}=\left\langle H_{1}, H_{2}\right\rangle, B_{0}=\left\langle H_{2}, H_{3}\right\rangle$, $C_{0}=\left\langle H_{3}, H_{4}\right\rangle, D_{0}=\left\langle H_{4}, H_{1}\right\rangle$ amalgamating $H_{2}, H_{3}, H_{4}, H_{1}$. Let $E=A_{0} *_{H_{2}} B_{0}$, $F=D_{0} *_{H_{4}} C_{0}$ and $H=H_{1} * H_{3}$. Then $E=H_{2} \times H, F=H_{4} \times H$ and $P_{0}=E *_{H} F=$ $\left(H_{2} * H_{4}\right) \times\left(H_{1} * H_{3}\right)$.

$(\Longrightarrow)$ Note that $H_{2} * H_{4}$ contains a free subgroup of rank 2 unless $\left|H_{2}\right|=2=\left|H_{4}\right|$ [10, p.195]. Similarly, $H_{1} * H_{3}$ contains a free subgroup of $\operatorname{rank} 2$ unless $\left|H_{1}\right|=$ $2=\left|H_{3}\right|$. Now if $P$ is $\mathcal{L E R F}$ then $P_{0}$ is $\mathcal{L E R \mathcal { F }}$. It follows from Lemma 3.1 that $\left|H_{2}\right|=2=\left|H_{4}\right|$ or $\left|H_{1}\right|=2=\left|H_{3}\right|$.

( $\Longleftarrow$ ) Assume that $\left|H_{1}\right|=2=\left|H_{3}\right|$. Then every subgroup of $H_{1} * H_{3}$ is f.g., hence, $H_{1} * H_{3} \cdot$ is $\mathcal{E R F}$. It follows from Theorem 1.3 that $P_{0}$ is $\mathcal{L} \mathcal{E R F}$. Note that $P=\left(\left(\left(P_{0} *_{A_{0}} A\right) *_{B_{0}} B\right) * C_{0} C\right) *_{D_{0}} D$. Since $A_{0}, B_{0}, C_{0}, D_{0}$ are finite, by Theorem $1.2, P$ is $\mathcal{L E R \mathcal { F }}$.

THEOREM 3.3. Let $P$ be the polygonal product of the four groups $A, B, C$, $D$, amalgamating the subgroups $H_{2}, H_{3}, H_{4}, H_{1}$, with trivial intersections, where the $H_{i}$ are contained in the centres of the vertex groups containing them. If $P$ is $\mathcal{L} \mathcal{E} \mathcal{R}$, then either $\left|H_{1}\right|=\left|H_{3}\right|=2$ or $\left|H_{2}\right|=\left|H_{4}\right|=2$.

Proof: If $P$ is $\mathcal{L E R \mathcal { F }}$, then the subgroup $P_{0}$ constructed in the proof of Theorem 3.2 is also $\mathcal{L E R \mathcal { F }}$. It follows that $\left|H_{1}\right|=\left|H_{3}\right|=2$, or $\left|H_{2}\right|=\left|H_{4}\right|=2$, as in the proof of Theorem 3.2 .

COROLLARY 3.4. Let $G$ be the polygonal product of the f.g. abelian groups $A$, $B, C, D$, amalgamating the subgroups $\langle b\rangle,\langle c\rangle,\langle d\rangle,\langle a\rangle$, with trivial intersections. If $G$ is $\mathcal{L E R F}$, then $|a|=2=|c|$ or $|b|=2=|d|$. In particular, the polygonal product of the four free abelian groups amalgamating the cyclic subgroups, with trivial intersections, is not $\mathcal{L E R F}$.

\section{REFERENCES}

[1] R.B.J.T. Allenby and R.J. Gregorac, 'On locally extended residually finite groups', in Lecture Notes in Mathematics 319, pp. 9-17 (Springer-Verlag, New York, 1973).

[2] R.B.J.T. Allenby and C.Y. Tang, 'The residual finiteness of some one-relator groups with torsion', J. Algebra 71 (1981), 132-140.

[3] R.B.J.T. Allenby and C.Y. Tang, 'On the residual finiteness of certain polygonal products', Canad. Math. Bull. 32 (1989), 11-17.

[4] G. Baumslag, 'On the residual finiteness of generalized free products of nilpotent groups', Trans. Amer. Math. Soc. 106 (1963), 193-209.

[5] A.M. Brunner, M.L. Frame, Y.W. Lee and N.J. Wielenberg, 'Classifying the torsion-free subgroups of the Picard group', Trans. Amer. Math. Soc. 282 (1984), 205-235.

[6] A. Karrass, A. Pietrowski and D. Solitar, 'The subgroups of polygonal products of groups', (Unpublished manuscript). 
[7] G. Kim, 'Conjugacy and subgroup separability of generalized free product', (Ph.D. thesis submitted to University of Waterloo, 1991).

[8] G. Kim, 'Cyclic subgroup separability of generalized free products', (Manuscript 1991).

[9] G. Kim and C.Y. Tang, 'On the residual finiteness of polygonal products of nilpotent groups', Canad. Math. Bull. (to appear).

[10] W. Magnus, A. Karrass and D. Solitar, 'Combinatorial Group Theory', in Pure and Applied Math. XIII (Wiley-Interscience, New York, London, Sydney, 1966).

[11] B.A. F. Wehrfritz, 'The residual finiteness of some generalized free products', J. London Math. Soc. 24 (1981), 123-126.

Department of Mathematics

Kangnung National University

Kangnung, Kangwon-Do, 210-702

Korea 